\title{
LOCAL STABILITY OF CONTINUOUS DYNAMICAL SYSTEMS IN PRESENCE OF NON-HYPERBOLIC EQUILIBRIA
}

\author{
F. BALIBREA \\ Department of Mathematics, University of Murcia, 30100 Murcia, Spain \\ E-mail: balibrea@um.es \\ A. MARTINEZ, J. C. VALVERDE \\ Department of Mathematics, University of Castilla-La Mancha, 02071 \\ Albacete, Spain \\ E-mail: Jose.Valverde@uclm.es
}

April 14, 2008

\begin{abstract}
In this work we analyze what happens when the generalized conditions given in [Balibrea et al., 2008], which produce the appearance of local bifurcations of continuous dynamical systems, fail. As a result, we are able to find out some situations of local stability in presence of non-hyperbolic equilibria.
\end{abstract}

\section{Introduction}

Consider a uniparametric family of continuous-time dynamical systems

$$
\dot{x}=f(x, \mu)
$$

where $\mu \in \mathbb{R}$ is the parameter and $x \in \mathbb{R}^{n}$ is the variable in the state space under consideration.

An experimental phenomenon which depends on a parameter could be represented by this family (see [Cugno \& Montrucchio, 1984]). Thus, we are interested in knowing if systems which are related in terms of the parameter, present similar dynamics.

Roughly speaking, if there is a qualitative change when crossing a particular value of the parameter $\mu_{0} \in \mathbb{R}$, then $\mu_{0}$ is said to be a bifurcation value or it is said that a bifurcation occurs at $\mu_{0}$. More rigorously, we can state the following definition.

Definition 1.1. A dynamical system $\left\{T, \mathbb{R}^{n}, \varphi\right\}$ is called locally topologically equivalent near an equilibrium $x_{0} \in \mathbb{R}^{n}$ to another dynamical system $\left\{T, \mathbb{R}^{n}, \psi\right\}$ near an equilibrium $y_{0} \in \mathbb{R}^{n}$ if there exists a homeomorphism $h: \mathbb{R}^{n} \rightarrow \mathbb{R}^{n}$ such that

i) h is defined in a small neighborhood $U \subset \mathbb{R}^{n}$;

ii) $h\left(x_{0}\right)=y_{0}$;

iii) h maps orbits of the first system in $U$ onto orbits of the second system in $h(U) \subset \mathbb{R}^{n}$, preserving the direction of time.

This definition let us assure that, in the neighborhoods $U$ and $h(U)$ the number and stability of the equilibria is the same. In other case, we have (locally) topologically non-equivalent systems

Definition 1.2. The appearance of a topologically non-equivalent system under variation of the parameter is called a bifurcation. 
In such a context, it is desirable to obtain a graphic which reflects this change of the topological type of the systems. This picture is called the bifurcation diagram.

Definition 1.3. A bifurcation diagram of the family is a stratification of its parameter space induced by the topological equivalence, together with representative phase portraits for each system.

An equilibrium $x \in \mathbb{R}^{n}$ for $f$ in $\operatorname{Diff}\left(\mathbb{R}^{n}\right)$ is called hyperbolic if the linearization $D f(x)$ has no eigenvalue equal to zero. From the theorem of Hartman-Grobman it follows (see [Guckenheimer \& Holmes, 1983] or [Nitecki, 1971]) that, to study local bifurcations of equilibria in parametric families $\dot{x}=f(x, \mu)$, it suffices to consider those parameters $\mu_{0}$ for which the corresponding system has a non-hyperbolic equilibrium $x_{0}$.

The simplest way in which the hyperolicity can be violated is when the real eigenvalue approaches zero as the parameter $\mu$ varies. However, not always the non-hyperbolicity implies the appearance of bifurcations and we are going to show how important the derivatives of the function $f$ are to maintain the structural stability.

So, we analyze some different phenomena which can occur when a non-hyperbolic equilibrium point with an eigenvalue equal to 0 exits. Sometimes a new kind of bifurcation (duplication of the standard one) occurs, although the common characteristic continues being the change in the number of equilibria when crossing the bifurcation value. But, sometimes, in spite of the existence of a non-hyperbolic point, the family will be (locally) structurally stable.

In the literature [Arrowsmith \& Place, 1990]; [Hale \& Koçak, 1991]; [Wiggins, 1990]), it is shown that under some nonzero conditions on the partial derivatives of $f$ up to the third order the standard bifurcations associated to the presence of an eigenvalue equals zero (saddle node, transcritical, pitchfork) appear.

In [Balibrea et al., 2008], we achive more general results assuring the presence of this bifurcations if those nonzero conditions are fulfilled by partial derivatives of higher order. As an extension of these results, in this paper we analyze what happens when any of the conditions given in [Balibrea et al., 2008] fails.

We call non-degenerate conditions to those statements which are sufficient to the appearance of a local bifurcation in a family with a non-hyperbolic equilibrium point. These conditions are given in terms of the partial derivatives of the function $f$. So, when any of these conditions fails, we will say that the condition is degenerated and the corresponding situation will be denominated degenerate followed by the name of the generic bifurcation. Thus, the purpose in the following three sections is to show what happens when any sufficient condition in the mentioned results is degenerated.

The main interest of it is that this analysis completes, to a certain extent, the study of the conditions related to the partial derivatives of the function $f$ and provides a method to conclude if a system presents a bifurcation and what kind of them is.

Without loss of generality, we shall assume that the bifurcation value is $\mu_{0}=0$ and the nonhyperbolic equilibrium is $x_{0}=0$. Hence, we shall give all our results under that assumption.

In addition, we shall suppose that the systems of the family are defined on $\mathbb{R}$ although, thanks to the Center Manifold Theory (e.g. see [Carr, 1981] or [Sijbrand, 1985]), they can also be used to describe these phenomena in uniparmetric families of systems defined on $\mathbb{R}^{n}$ or, more generally, on a Banach space.

\section{Degenerate Fold Bifurcation}

In [Balibrea et al., 2008], the sufficient conditions which produce a standard fold bifurcation are generalized, and renamed non-degenerate conditions. In particular, the following theorem is achieved:

Theorem 2.1. Suppose that a one-parameter family of systems $\dot{x}=f(x, \mu)$, with $f$ belonging to class $C^{2 n}$, has at $\mu_{0}=0$ the equilibrium $x_{0}=0$ and let $f_{x}(0,0)=0$.

Assume that the following non-degenerate conditions are satisfied:

$$
\begin{aligned}
& \text { ( } \left.\mathrm{F}_{1}\right) f_{x x}(0,0)=f_{x x x}(0,0)=\cdots=f_{x^{2 n-1}}(0,0)=0 \\
& f_{x^{2 n}}(0,0) \neq 0
\end{aligned}
$$


$\left(\mathrm{F}_{2}\right) f_{\mu}(0,0) \neq 0$.

\section{Then the family undergoes a fold bifurcation.}

Then, it is natural to wonder what happens if any of the above conditions in Theorem 2.1 changes. In the following theorem we are considering changes in the condition $\left(F_{1}\right)$.

Theorem 2.2. Suppose that a one-parameter family of systems $\dot{x}=f(x, \mu)$, with $f$ belonging to class $C^{2 n+1}$, has at $\mu_{0}=0$ the equilibrium $x_{0}=0$ and let $f_{x}(0,0)=0$.

Assume that the following non-degenerate conditions are satisfied:

$\left(\mathrm{F}_{1}\right) f_{x x}(0,0)=f_{x x x}(0,0)=\cdots=f_{x^{2 n}}(0,0)=0$, $f_{x^{2 n+1}}(0,0) \neq 0$

$\left(\mathrm{F}_{2}\right) f_{\mu}(0,0) \neq 0$.

Then the family does not undergo a fold bifurcation. Moreover, all the maps corresponding to small values of $\mu$, present a unique fixed point with the same type of stability.

Proof. Thanks to the hypotheses, we have:

(a) $f(0,0)=0$

(b) $f_{x}(0,0)=0$

(c) $f_{x x}(0,0)=\cdots=f_{x^{2 n}}(0,0)=0, \quad f_{x^{2 n+1}}(0,0) \neq 0$

(d) $f_{\mu}(0,0) \neq 0$

Because of (a) and (d), the Implicit Function Theorem assures the existence of a unique $C^{2 n+1}$ curve $\mu(x)$ for $x$ near zero with:

$$
\left\{\begin{array}{l}
\mu(0)=0 \\
f(x, \mu(x))=0
\end{array}\right.
$$

Differentiating the last equation and evaluating (each obtained equation) at $x=0$ successively, we will find:

$\frac{d \mu}{d x}(0)=\frac{d^{2} \mu}{d x^{2}}(0)=\cdots=\frac{d^{2 n-1} \mu}{d x^{2 n}}(0)=0, \frac{d^{2 n+1} \mu}{d x^{2 n+1}}(0) \neq 0$

So $\mu(x)$ has neither a maximum at $x=0$ nor a minimum. It means that the curve crosses the line $\mu=0$ in the $x, \mu$-plane and therefore the existence and uniqueness of the equilibria is proved.
For the stability of this equilibria, we consider the equation which define them:

$$
f(x, \mu(x))=0
$$

Now, differentiating it, we obtain

$$
f_{x}+f_{\mu} \frac{d \mu}{d x}=0
$$

or in other words

$$
f_{x}=-f_{\mu} \frac{d \mu}{d x}
$$

In each case, one can deduce the sign of $f_{\mu} \frac{d \mu}{d x}$ in terms of the signs of $f_{x^{2 n+1}}(0,0)$ and $f_{\mu}$. Effectively, in view of the value of the derivatives of $\mu(x)$, it follows that, in a small neighborhood of $x=0$, its McLaurin series is

$$
\mu(x)=\frac{1}{(2 n+1) !} \frac{d^{2 n+1} \mu}{d x^{2 n+1}}(0) x^{2 n+1}+\mathcal{O}\left(x^{2 n+2}\right)
$$

Thus, $\frac{d \mu}{d x}$ has constant sign for all $x$ in a neighboorhood of the origin, and this sign is the opposite to the one of $f_{x^{2 n+1}}(0,0)$.

This fact let us conclude that when $f_{x^{2 n+1}}(0,0)$ and $f_{\mu}(0,0)$ have opposite signs, the equilibria of the family are stable; and when $f_{x^{2 n+1}}(0,0)>0$ and $f_{\mu}(0,0)$ have the same, sign the equilibria are unstable.

Remark 2.3. Theorem 2.2 let us deduce that, in spite of having a non-hyperbolic equilibrium, the family is locally structurally stable.

Now we are considering changes in the condition $\left(\mathrm{F}_{2}\right)$. When this condition fails the situation is more involved. In particular, if the family is of the form

$$
f(x, \mu)= \pm \mu^{2 m+1} \pm x^{2 n}, m \geq 0, n \geq 1, m, n \in \mathbb{Z}
$$

it still undergoes a fold bifurcation when the parameter value $\mu=0$ is crossed. But, if it has a expression such as

$$
f(x, \mu)= \pm \mu^{2 m+2} \pm x^{2 n}, m \geq 0, n \geq 1, m, n \in \mathbb{Z},
$$

then one of the situations which occurred on one side of $\mu=0$ for the fold bifurcation, occurs now on 
4 J.C. Valverde et al.

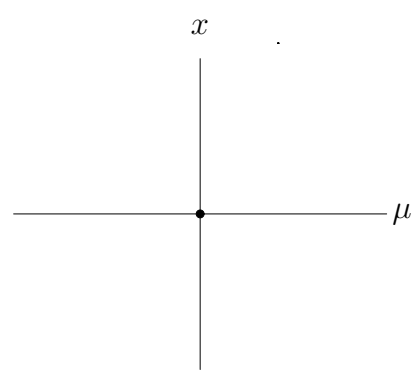

$f(x, \mu)=\mu^{2 m+2}+x^{2 n}$

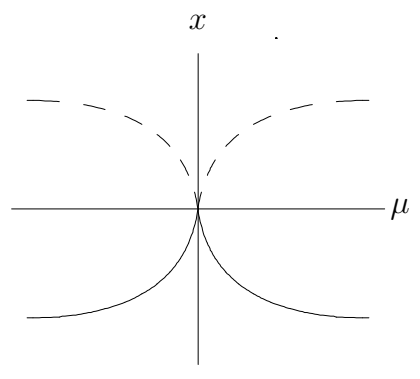

$f(x, \mu)=-\mu^{2 m+2}+x^{2 n}$

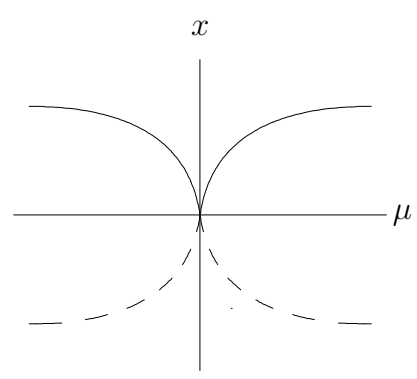

$f(x, \mu)=\mu^{2 m+2}-x^{2 n}$

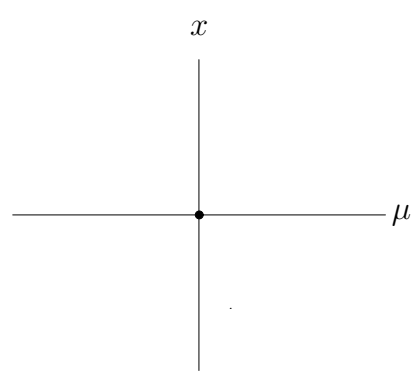

$f(x, \mu)=-\mu^{2 m+2}-x^{2 n}$

Fig. 1. Bifurcation diagrams of degenerate fold bifurcations. 
both sides. In this case, we have the four bifurcation diagrams of Figure 1, where we have supposed that $n>m+1$. In the other cases, the qualitative behavior as the parameter varies is similar.

However, the situation can change if we introduce terms of lower order including the parametric variable in the previous expressions. This is the case of the family

$$
f(x, \mu)=\mu x+\mu^{3}+x^{2}
$$

which does not undergo a fold bifurcation; on the contrary, two equilibria appear in both sides of $\mu=$ 0 .

Nevertheless, bifurcation diagrams are not entirely chaotic and many bifurcation diagrams of different families can look similar. Equivalent bifurcation diagrams are obtained if we have (locally) topologically equivalent families what means the following

Definition 2.4. Two one-parameter families of continuous-time dynamical systems $\dot{x}=f(x, \mu)$ and $\dot{y}=g(y, \nu)$ with $x, y \in \mathbb{R}^{n}$ and $\mu, \nu \in \mathbb{R}$ are called locally topologically equivalent near the origin, if there exists a map

$$
(x, \mu) \rightarrow\left(h_{\mu}(x), p(\mu)\right)
$$

defined in a small neighborhood of $(x, \mu)=(0,0)$ such that

1. $p$ is homeomorphism defined in a small neighborhood of $\mu=0$, and $p(0)=0$;

2. $h_{\mu}$ is a parameter-dependent homeomorphism defined in a small neighborhood $U_{\mu}$ of $x=0$, verifying $h_{0}(0)=0$ and mapping orbits of the first system in $U_{\mu}$ onto orbits of the second systems in $h_{\mu}\left(U_{\mu}\right)$, preserving the direction of time.

So, at this point, we can state that if we suppose that a family $g: \mathbb{R} \times \mathbb{R} \rightarrow \mathbb{R}$ is induced by a family $f: \mathbb{R} \times \mathbb{R} \rightarrow \mathbb{R}$ which undergoes a fold bifurcation, i.e., $g(x, \mu)=f(x, p(\mu))$, where $p: \mathbb{R} \rightarrow \mathbb{R}$ is an homeomorphism such that $p(0)=0$, then, due to the correspondence between systems of the family $g$ with systems of the family $f$, for small values of the parameter, one can deduce that $g$ undergoes a fold bifurcation. On the contrary, if $f$ is as in the examples above, then one of the situations which occurred on one side of $\mu=0$ for a fold bifurcation, happens to $g$ on both sides of $\mu=0$.

When a family verifies $\left(\mathrm{DF}_{1}\right)$, the changes in the condition associated to the derivatives with respect to the parameter can also provide a variety of different situations depending on the nonzero such derivatives. But, if $g: \mathbb{R} \times \mathbb{R} \rightarrow \mathbb{R}$ is induced by a family $f \mathbb{R} \times \mathbb{R} \rightarrow \mathbb{R}$ which satisfies the conditions of Theorem 2.2, then we have local structural stability because of the topological equivalence of both families.

\section{Degenerate Transcritical Bifurcation}

As in the above case, in [Balibrea et al., 2008], the sufficient conditions to the standard transcritical bifurcation occurs are generalized and renamed nondegenerate conditions. In this sense, the following theorem is achived.

Theorem 3.1. Suppose that a one-parameter family of systems $\dot{x}=f(x, \mu)$, with $f$ belonging to class $C^{2 n+1}$, has at $\mu_{0}=0$ the equilibrium $x_{0}=0$ and let $f_{x}(0,0)=0, f_{\mu}(0,0)=0$.

Assume that the following non-degenerate conditions are satisfied:

$$
\begin{aligned}
& \left(\mathrm{T}_{1}\right) f_{x x}(0,0)=f_{x x x}(0,0)=\cdots=f_{x^{2 n-1}}(0,0)=0 \\
& f_{x^{2 n}}(0,0) \neq 0 \\
& \left(\mathrm{~T}_{2}\right) f_{x \mu}(0,0) \neq 0 .
\end{aligned}
$$

Then the family undergoes a transcritical bifurcation.

In this context, the following question arises: what happens if any of these non-degenerate conditions changes?

First of all, we must observe that the change in the condition $\left(\mathrm{T}_{1}\right)$ provides a family with a pitchfork bifurcation. Hence, we only analyze the change in $\left(\mathrm{T}_{2}\right)$.

When the condition $\left(T_{2}\right)$, concerning the derivatives with respect to the parameter, fails, a proof similar to that made for Theorem 3.1 does not work, since we can not apply the Implicit Function Theorem in the same way. However, one can easily 
check that for particular families such as

$f(x, \mu)= \pm \mu^{2 m+1} x \pm x^{2 n}, m \geq 0, n \geq 1, m, n \in \mathbb{Z}$

a transcritical bifurcation also appears and for others such as

$f(x, \mu)= \pm \mu^{2 m+2} x \pm x^{2 n}, m \geq 0, n \geq 1, m, n \in \mathbb{Z}$

one of the situations, which occurred on one side of $\mu=0$ for the transcritical bifurcation, occurs now on both sides, giving the four bifurcation diagrams of Figure 2, where, as before, we have supposed that $n>m+1$.

More generally, as in the fold case, if we suppose that a family $\dot{x}=g(x, \mu)$ is induced by a family $\dot{x}=f(x, \mu)$ which undergoes a transcritical bifurcation, by means of a homeomorphism $p$, then, because of the correspondence between functions of the family $g$ with functions of the family $f$ for small values of the parameter, one can deduce that $g$ undergoes a transcritical bifurcation. On the contrary, if $f$ is as in the examples above, then one of the situations which occurred on one side of $\mu=0$ for a transcritical bifurcation, happens to $g$ on both sides of $\mu=0$.

On the other hand, observe that the change in both non-degenerated conditions corresponds to the change in the second non-degenerated condition of Theorem 4.1 that we are going to analyze in the next section.

\section{Degenerate Pitchfork Bifurcation}

We are going to study analogous situations to those above, based on the following result, which one can see in [Balibrea et al., 2008], too.

Theorem 4.1. Suppose that a one-parameter family of systems $\dot{x}=f(x, \mu)$, with $f$ belonging to class $C^{2 n+2}$, has at $\mu_{0}=0$ the equilibrium $x_{0}=0$ and let $f_{x}(0,0)=0, f_{\mu}(0,0)=0$.

Assume that the following non-degenerate conditions are satisfied:

$\left(\mathrm{P}_{1}\right) f_{x x x}(0,0)=f_{x^{4}}(0,0)=\cdots=f_{x^{2 n-1}}(0,0)=0$, $f_{x^{2 n+1}}(0,0) \neq 0$

$\left(\mathrm{P}_{2}\right) f_{x \mu}(0,0) \neq 0$.
Then the family undergoes a pitchfork bifurcation.

When we study the pitchfork bifurcation in the literature, one of the natural imposed conditions to the function $f$ is to be odd with respect to $x$. However, it is not necessary as one can see in Theorem 4.1. So, if we assume the mentioned natural imposed condition, we could not consider the change in the first non-degenerate condition, because all the even derivatives with respect to $x$ evaluated at the origin would be zero. But, if we suppress that the family must be odd, then that change provides a family with a transcritical bifurcation.

As in the transcritical case, when the condition $\left(\mathrm{P}_{2}\right)$, concerning the derivatives with respect to the parameter, fails, a proof similar to that one made for the previous theorem does not work. However, one can easily check that for particular families such as

$$
f(x, \mu)= \pm \mu^{2 m+1} x \pm x^{2 n+1}, m \geq 0, n \geq 1, m, n \in \mathbb{Z}
$$

a pitchfork bifurcation appears, while for others such as

$$
f(x, \mu)= \pm \mu^{2 m+2} x \pm x^{2 n+1}, m \geq 0, n \geq 1, m, n \in \mathbb{Z}
$$

one of the situations, which occurred on one side of $\mu=0$ for the pitchfork bifurcation, now occurs on both sides, giving the four bifurcation diagrams in Figure 3, where we have supposed that $2 n+1>$ $2 m+2$. The qualitative behavior of the family in the other case is similar.

As in the previous cases, one can obtain similar results for families induced by one which satisfies the conditions mentioned in the theorem 4.1, by means of a homeomorphism $p$ defined for values of $\mu$ sufficiently small.

Once more, we observe that the change in both non-degenerate conditions corresponds to the change in the second one of Theorem 3.1 what was studied in the previous section.

\section{Acknowledgements}

This research has been partially supported by the Regional Grant PAI-06-0125 and the Spanish National Grant MTM2005-03868. 

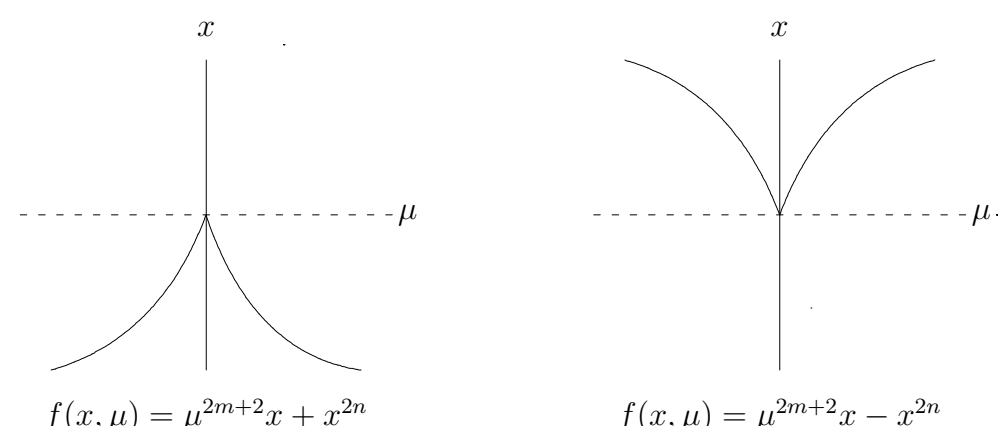

$$
f(x, \mu)=\mu^{2 m+2} x+x^{2 n}
$$

$$
f(x, \mu)=\mu^{2 m+2} x-x^{2 n}
$$
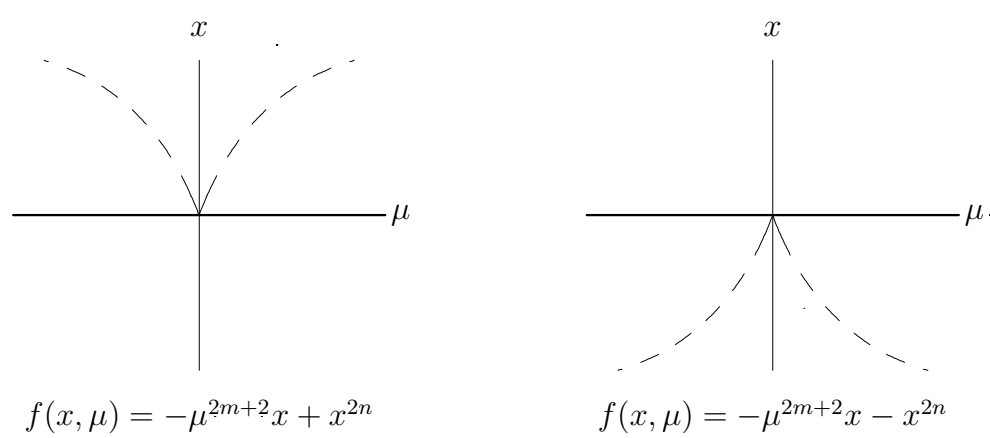

Fig. 2. Bifurcations diagrams of degenerated transcritical bifurcations. 
8 J.C. Valverde et al.
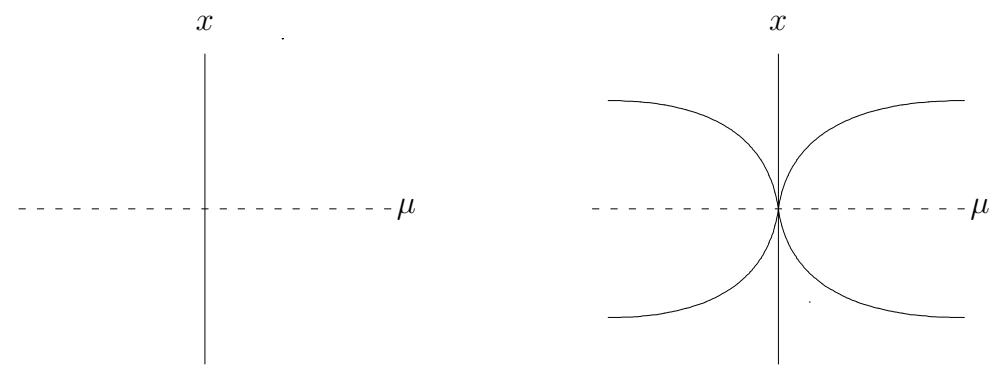

$f(x, \mu)=\mu^{2 m+2} x+x^{2 n+1}$

$f(x, \mu)=\mu^{2 m+2} x-x^{2 n+1}$
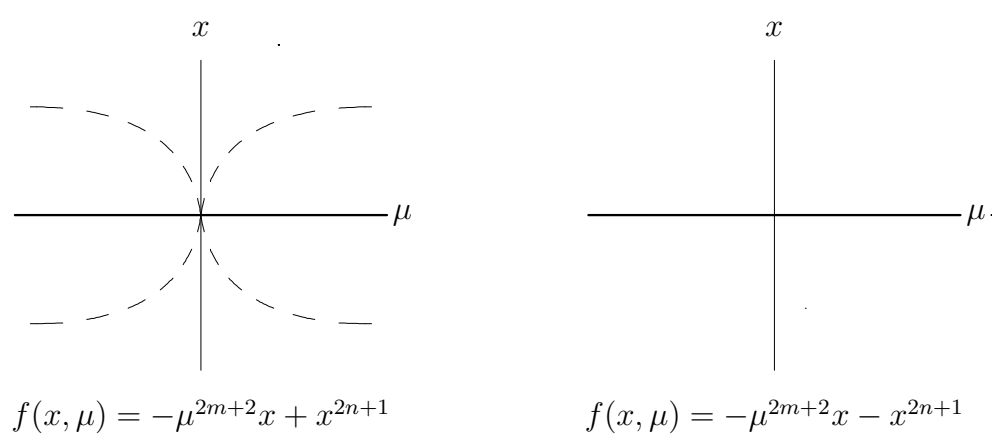

Fig. 3. Bifurcation diagrams of degenerate pitchfork bifurcations. 


\section{References}

Arrowsmith, D. K. \& Place, C. M. [1990] An Introduction to Dynamical Systems (Cambridge University Press, Cambridge).

Balibrea, F., Martinez, A. \& Valverde, J.C. [2008] "Local bifurcations of one-dimensional continuous dynamical systems under higher order conditions," Acta. Math. Sin.-English Ser., (to appear).

Carr J. [1981] Applications of Center Manifold Theory (Springer-Verlag, New York, Heidelberg, Berlin).

Chow, S. N., Li, C. \& Wang, D. [1994] Normal Forms and Bifurcations of Planar Vector Fields (Cambridge Univ. Press, Cambridge).

Chua, L. O. \& Kokubu, H. [1989] "Normal forms for nonlinear vector fields II. Applications," IEEE Trans. Circuits and Systems 36, 51-70.

Cugno, F. \& Montrucchio, L. [1984] Some New Techniques for Modelling Nonlinear Economic Fluctuations: A Brief Survey, in Nonlinear Models of Fluctuating Growth (Springer-Verlag, New York).

Guckenheimer, J. \& Holmes, P. [1983] Nonlinear Oscillations, Dynamical Systems and Bifurcations of Vector Fields (Springer-Verlag, New York, Heidelberg, Berlin).

Hale, J. \& Koçak, H. [1991] Dynamics and Bifurcations (Springer-Verlag, New York, Heidelberg, Berlin).

Kuznetsov, Y. A. [2004] Elements of Applied Bifurcation Theory, 3rd Edition (Springer-Verlag, New York).

Nayfeh, A. H. [1993] Methods of Normal Forms (John Wiley, New York).

Nitecki, Z. [1971] Differentiable Dynamics (M.I.T. Press, Cambridge).

Sijbrand, J. [1985] "Properties of center manifolds," Trans. Amer. Math. Soc. 289, 431-469.

Takens, F. [1974] "Singularities of vector fields," Publ. Math. Inst. Hautes Ėtudes Sci. 43, 47-100.
Ushiki, S. [1984] "Normal forms for singularities of vector fields," Jpn. J. Appl. Math. 1, 1-34.

Wang, Z.G. [2007] "Normal form for families of Hamiltonian systems," Acta Math. Sin. (Engl. Ser.) 23(7), 1199-1216.

Wiggins, S. [1990] Introduction to Applied Nonlinear Systems and Chaos (Springer-Verlag, New York). 